\title{
Off-shell initial state effects in Drell-Yan process at hadron colliders
}

\author{
Maxim Nefedov $^{1, *}$ and Vladimir Saleev ${ }^{1, * *}$ \\ ${ }^{1}$ Samara National Research University, Moskovskoe Shosse 34, 443086 Samara, Russia
}

\begin{abstract}
We discuss production of Drell-Yan lepton pairs at hadron colliders in the framework of the parton Reggeization approach, which includes off-shell initial state effects in a gauge-invariant way. We have compared our numerical results for $q_{T}$-spectra of the lepton pair with the experimental data, obtained by E-288 collaboration ( $\sqrt{S}=19.4$ and $23.8 \mathrm{GeV}$ ) and found a good agreement. We have also made predictions for the Drell-Yan structure functions at NICA pp-collider $(\sqrt{S}=24 \mathrm{GeV})$.
\end{abstract}

\section{Introduction}

The Drell-Yan (DY) process of production of lepton pairs with large invariant mass in hadronic collisions is one of the most important test of perturbative quantum chromodynamics (QCD), as well as the unique source of information about partonic structure of hadrons. Apart from the inclusive cross-section, differential w.r.t. squared invariant mass $\left(Q^{2}\right)$, transverse momentum $\left(q_{T}\right)$ and rapidity $(Y)$ of the lepton pair or some equivalent variable, such as momentum fraction in the Collinear Parton model (CPM) $\left(x_{A, B}\right)$, also structure functions or angular coefficients, which parameterize the angular distribution of leptons in the rest frame of the lepton pair are often under consideration. Behavior of the latter class of observables in the region of relatively small $q_{T} \leq Q$ will be the main subject of the present paper.

At low $q_{T} \ll Q$, already the prediction of inclusive cross-section, integrated over all directions of lepton momentum in the center-of-mass frame of the pair, presents a considerable difficulty for the conventional CPM, since at any fixed order of perturbation theory the cross-section diverges as $1 / q_{T}^{2}$ at $q_{T} \rightarrow 0$. These un-physical divergence is regulated through the resummation of higher-order corrections in $\alpha_{s}$ enhanced by $\log ^{2}\left(q_{T} / Q\right)$ and $\log \left(q_{T} / Q\right)$ through Collins-Soper-Sterman formalism [1], which later has been reformulated in a form of Transverse Momentum Dependent (TMD) factorization theorem [2].

In TMD-factorization, the hard-scattering coefficient (HSC) does not depend explicitly on the transverse momenta of colliding partons. Instead, it is calculated with on-shell initial-state partons and the corresponding partonic tensor automatically satisfies the QED Ward identity. However, it is possible to develop a complementary approach to TMD factorization, starting not from the collinear limit but from Multi-Regge limit for QCD scattering amplitudes, i.e. from the limit when all final-state particles are highly-separated in rapidity. We call this scheme of calculations - the Parton Reggeization Approach (PRA).

\footnotetext{
*e-mail: nefedovma@gmail.com

**e-mail: saleev@samsu.ru
} 
In PRA, the HSC, although being gauge-invariant, nevertheless explicitly depends on the transverse momenta of initial-state partons. Below we demonstrate, that this dependence is important to calculate the angular structure functions.

\section{Parton Reggeization Approach}

A more detailed introduction to the PRA and derivation of our factorization formula is presented in [3]. Here we only briefly outline the main ideas. Factorization formula of PRA is based on modified-MRK approximation for QCD matrix elements. This approximation smoothly interpolates between the well-known collinear and Multi-Regge asymptotics (see e.g. [4] for the review of the latter) of the matrix element of ordinary CPM hard subprocess with emission of two additional partons. In the collinear limit, additional partons have $\left|\mathbf{k}_{T}\right| \ll \mu$ where $\mu$ is the hard scale ( $\mu \sim Q$ in the case of DY process), while in the MultiRegge limit, additional partons are highly separated in rapidity from the system of interest $\left(l^{+} l^{-}\right.$for DY process), while their typical $\left|\mathbf{k}_{T}\right| \sim \mu$. In both limits, QCD matrix element can have the $t$-channel-factorized form, however in the MRK case the partons, propagating in the $t$-channels are not ordinary QCD quarks and gluons, but special gauge-invariant degrees of freedom of high-energy QCD, called Reggeized quarks $(Q)$ and gluons $(R)$. Due to the $t$-channel-factorized form of the mMRK-approximation, the cross section of lepton pair production in proton-proton collisions, $p\left(P_{1}\right)+p\left(P_{2}\right) \rightarrow l^{+}\left(k_{1}\right)+l^{-}\left(k_{2}\right)+X$, can be presented in $k_{T}$-factorized form:

$$
d \sigma=\int_{0}^{1} \frac{d x_{1}}{x_{1}} \int \frac{d^{2} \mathbf{q}_{T 1}}{\pi} \Phi_{q}\left(x_{1}, t_{1}, \mu^{2}\right) \int_{0}^{1} \frac{d x_{2}}{x_{2}} \int \frac{d^{2} \mathbf{q}_{T 2}}{\pi} \Phi_{\bar{q}}\left(x_{2}, t_{2}, \mu^{2}\right) \cdot d \hat{\sigma}_{\mathrm{PRA}},
$$

where $x_{1}=q_{1}^{+} / P_{1}^{+}, x_{2}=q_{2}^{-} / P_{2}^{-}$, four-momenta of partons in the initial-state of the leading order (LO) PRA hard-scattering subprocess $Q\left(q_{1}\right)+\bar{Q}\left(q_{2}\right) \rightarrow l^{+}+l^{-}$are parameterized as $q_{1}=\frac{1}{2} q_{1}^{+} n_{-}+q_{T 1}, q_{2}=\frac{1}{2} q_{2}^{-} n_{+}+q_{T 2}, t_{1,2}=\mathbf{q}_{T 1,2}=-q_{1,2}^{2}$, and light-cone vectors are defined as $n_{-}^{\mu}=2 P_{1}^{\mu} / \sqrt{S}, n_{+}^{\mu}=2 P_{2}^{\mu} / \sqrt{S}$ where $S=\left(P_{1}+P_{2}\right)^{2}=2 P_{1} P_{2}$. For any four-vector the light-cone components are $k^{ \pm}=\left(k n^{ \pm}\right)$, so that $k^{2}=k^{+} k^{-}-\mathbf{k}_{T}^{2}$, and we do not distinguish between the upper and lower light-cone indices $k^{ \pm}=k_{ \pm}$.

The partonic cross-section $d \hat{\sigma}_{\mathrm{PRA}}$ is:

$$
d \hat{\sigma}_{\mathrm{PRA}}=\frac{\overline{\left|\mathcal{A}_{P R A}\right|^{2}}}{2 S x_{1} x_{2}} \cdot(2 \pi)^{4} \delta^{(4)}\left(q_{1}+q_{2}-k_{1}-k_{2}\right) d \Phi\left(k_{1}, k_{2}\right),
$$

where $d \Phi\left(k_{1}, k_{2}\right)$ is the element of Lorentz-invariant phase space for final-state leptons, $2 x_{1} x_{2} S$ is the appropriate flux-factor for the initial state off-shell partons (see discussion in [3]).

The LO unintegrated PDF (unPDF) $\Phi_{q, \bar{q}}\left(x_{1,2}, t_{1,2}, \mu^{2}\right)$ in Eq. 1 is related with ordinary PDFs of CPM as follows:

$$
\Phi_{q}\left(x, t, \mu^{2}\right)=\frac{T_{q}\left(t, \mu^{2}\right)}{t} \times \frac{\alpha_{s}(t)}{2 \pi} \int_{x}^{1-\Delta} d z \frac{x}{z}\left[P_{q q}(z) f_{q}\left(\frac{x}{z}, \mu^{2}\right)+P_{q g}(z) f_{g}\left(\frac{x}{z}, \mu^{2}\right)\right],
$$

where $f_{q, g}\left(x, \mu^{2}\right)$ are relevant collinear PDFs, and the Kimber-Martin-Ryskin cut condition [5, 6], $\Delta=\frac{\sqrt{t}}{\sqrt{\mu^{2}}+\sqrt{t}}$, follows from the rapidity ordering between the last emission and the hard subprocess. In Eq. (3), $T_{q}\left(t, \mu^{2}\right)$ is well known Sudakov factor with boundary conditions 
$T_{q}\left(\mu^{2}, \mu^{2}\right)=0$ and $T_{q}\left(\mu^{2}, \mu^{2}\right)=1$, which lead to the following normalization for unintegrated PDF: $\int_{0}^{\mu^{2}} d t \Phi_{q}\left(x, t, \mu^{2}\right)=x f_{q}\left(x, \mu^{2}\right)$.

In the PRA, the squared amplitude of the subprocess $\left(Q \bar{Q} \rightarrow l^{+} l^{-}\right)$can be presented as convolution of standard lepton tensor $L^{\mu v}=2\left[-Q^{2} g^{\mu \nu}+2\left(k_{1}^{\mu} k_{2}^{v}+k_{1}^{v} k_{2}^{\mu}\right)\right]$ and the partonic tensor $w_{\mu \nu}^{\mathrm{PRA}}$ :

$$
\overline{\left|\mathcal{A}\left(Q \bar{Q} \rightarrow l^{+} l^{-}\right)\right|^{2}}=\frac{16 \pi^{2}}{N_{c} Q^{4}} \alpha^{2} e_{q}^{2} L^{\mu \nu} w_{\mu \nu}^{\mathrm{PRA}},
$$

where $N_{c}=3$ and partonic tensor reads

$$
w_{\mu \nu}^{\mathrm{PRA}}=\frac{1}{4} \operatorname{tr}\left[\left(\frac{q_{2}^{-}}{2} \hat{n}^{+}\right) \Gamma_{\mu}\left(q_{1}, q_{2}\right)\left(\frac{q_{1}^{+}}{2} \hat{n}^{-}\right) \Gamma_{\nu}\left(q_{1}, q_{2}\right)\right],
$$

where factor $1 / 4$ stands for averaging over spins of quark and antiquark, $\hat{k}=k_{\mu} \gamma^{\mu}$ and $\Gamma_{\mu}\left(q_{1}, q_{2}\right)$ is the Fadin-Sherman $Q \bar{Q} \gamma$ vertex [7, 8]:

$$
\Gamma_{\mu}\left(q_{1}, q_{2}\right)=\gamma_{\mu}-\hat{q}_{1} \frac{n_{\mu}^{-}}{q_{2}^{-}}-\hat{q}_{2} \frac{n_{\mu}^{+}}{q_{1}^{+}} .
$$

The QED Ward identity $\left(q_{1}+q_{2}\right)^{\mu} \Gamma_{\mu}\left(q_{1}, q_{2}\right)=0$ is satisfied by this vertex for any $q_{1}$ and $q_{2}$.

The first term in Eq. (5) corresponds to the usual $t$-channel quark-antiquark annihilation diagram (a) in the Fig. 1. While other two ("eikonal") terms in Eq. (5), contain factors $1 / q_{1}^{+}$and $1 / q_{2}^{-}$. These factors can be understood as remnants of $s$-channel propagators in the diagrams where photon interacts with particles highly separated in rapidity from the lepton pair. More rigorously, the common lore in high-energy QCD (see e.g. [4, 9] and references therein) is, that particles in the central rapidity region interact with other particles, highly separated from them in rapidity, as with Wilson lines stretched along the light-cone. The "eikonal" terms in Eq. (5) correspond to the coupling of photon with these Wilson lines. Corrections to this approximation are suppressed by powers of $e^{-\Delta y}$, where $\Delta y$ is the rapidity gap. In other words, inclusion of the second and third terms in Eq. (5) is the simplest possible way to effectively take into account the diagrams (b) and (c) in Fig. 1, where photon interacts directly with the proton and its remnants. This approximation assumes only that the systems $X_{1}$ and $X_{2}$ are highly separated in rapidity from the central region. Rapidity gap between collinear subgraphs exists for $q_{T} \ll Q$ at the level of leading region for the Drell-Yan process. This rapidity gap is filled by soft particles emitted from the Glauber gluon exchanges between collinear subgraphs, which does not lead to violation of factorization (See e.g sec. 14.2 and 14.3 in [2]).

\section{Structure functions for DY process in PRA}

In the notation of [10] the differential cross section of DY pair production in collision of non-polarized protons can be written as the combination of helicity structure functions (SFs):

$$
\begin{aligned}
\frac{d \sigma}{d x_{A} d x_{B} d^{2} \mathbf{q}_{T} d \Omega} & =\frac{\alpha^{2}}{4 Q^{2}}\left[F_{U U}^{(1)} \cdot\left(1+\cos ^{2} \theta\right)+F_{U U}^{(2)} \cdot\left(1-\cos ^{2} \theta\right)+\right. \\
& \left.+F_{U U}^{(\cos \phi)} \cdot \sin (2 \theta) \cos \phi+F_{U U}^{(\cos 2 \phi)} \cdot \sin ^{2} \theta \cos (2 \phi)\right]
\end{aligned}
$$

were $x_{A, B}=Q e^{ \pm Y} / \sqrt{S}$, angles $\theta$ and $\phi$ are defined in the Collins-Soper frame [11] and $F_{U U}^{1,2, \cos 2 \phi}$ are the helicity SFs at some fixed values of $S, q_{T}=\left|\mathbf{q}_{T 1}+\mathbf{q}_{T 2}\right|, x_{A}, x_{B}$. By means of 


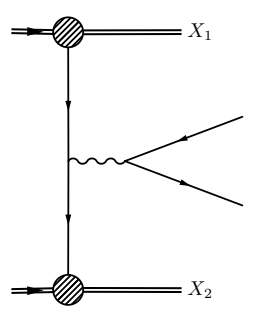

(a)

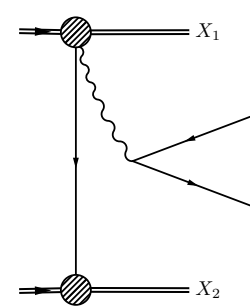

(b)

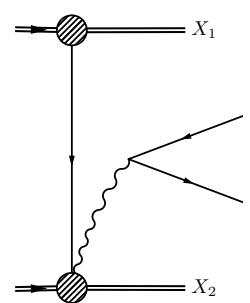

(c)

Figure 1. Feynman diagrams for the $t$-channel quark-antiquark annihilation subprocess (a), which leads to the usual parton-model picture, and direct interaction subprocesses $(b, c)$ which are necessary to restore QED gauge invariance of diagram (a)

factorization formula (1) SFs can be represented as:

$$
F_{U U}^{(1, \ldots)}=\frac{S}{6 \pi^{2} Q_{T}^{4}} \int d t_{1} \int d \phi_{1} \sum_{q} \Phi_{q}^{p}\left(x_{1}, t_{1}, \mu^{2}\right) \Phi_{\bar{q}}^{p}\left(x_{2}, t_{2}, \mu^{2}\right) \cdot e_{q}^{2} f^{(1, \ldots)},
$$

where $t_{2}=\left(\mathbf{q}_{T}-\mathbf{q}_{T 1}\right)^{2}, Q_{T}^{2}=Q^{2}+q_{T}^{2}$ and $e_{q}$ is the quark electric charge in units of electron charge. Projecting the partonic tensor (4) on transverse, longitudinal, single spin-flip and double spin-flip helicity states of the virtual photon, one obtains the following expressions for partonic SFs in PRA [12]:

$$
\begin{array}{r}
f_{\mathrm{PRA}}^{(1)}=Q^{2}+\frac{q_{T}^{2}}{2}, f_{\mathrm{PRA}}^{(2)}=\left(\mathbf{q}_{T 1}-\mathbf{q}_{T 2}\right)^{2}, \\
f_{\mathrm{PRA}}^{(\cos \phi)}=\sqrt{\frac{Q^{2}}{q_{T}^{2}}}\left(\mathbf{q}_{T 1}^{2}-\mathbf{q}_{T 2}^{2}\right), f_{\mathrm{PRA}}^{(\cos 2 \phi)}=\frac{q_{T}^{2}}{2} .
\end{array}
$$

In the case of collisions of identical target and projectile (e.g. in $p p$-collisions), the SF $F_{U U}^{\cos \phi}$ is equal to zero in PRA, due to the factor $\left(t_{1}-t_{2}\right)$ in Eq. 7. However, for collisions of different particles we expect nonzero value of $F_{U U}^{\cos \phi}$ due to the difference of transversemomentum distributions of quarks and antiquarks in the projectile and on the target.

\section{Numerical results and discussion}

To justify the use of PRA at relatively low $\sqrt{S}=24 \mathrm{GeV}$, which is expected to be achieved during the operation of NICA collider in the $p p$-collider mode (see e.g. [13]), we compare our numerical results for the differential cross-section $E d \sigma / d^{3} \mathbf{q}$ as a function of $q_{T}, Y$ and $Q$ with the experimental data of E-288 Collaboration [14], obtained in the collisions of the proton beam with the platinum fixed target at $\sqrt{S}=19.4$ and $23.8 \mathrm{GeV}$ (Fig. 2). The KMR unPDF is generated from the LO PDFs MSTW-2008 [15]. We use the factorization scalechoice $\mu_{F}=\xi Q_{T}$ and vary $\xi$ in the range $1 / 2 \leq \xi \leq 2$ to obtain the scale-uncertainty band. The " $\pi^{2}$-resummation" K-factor (see Eq. (53) in [12]) is applied to the cross-section. From Figs. 2 and 3 one can see, that LO PRA calculation describes the E-288 data at all values of $q_{T}, Q$ and $Y$ reasonably well.

Comparison of LO PRA predictions for the $q_{T}$-dependence of polarization parameters $\lambda$ and $v$ with the experimental data of NuSea Collaboration [16] obtained in the $p p$-collisions with $\sqrt{S}=39 \mathrm{GeV}$ is presented in [12] and also demonstrates a good agreement with data. This agreement justifies our attempt to provide the predictions for helicity SFs below. 

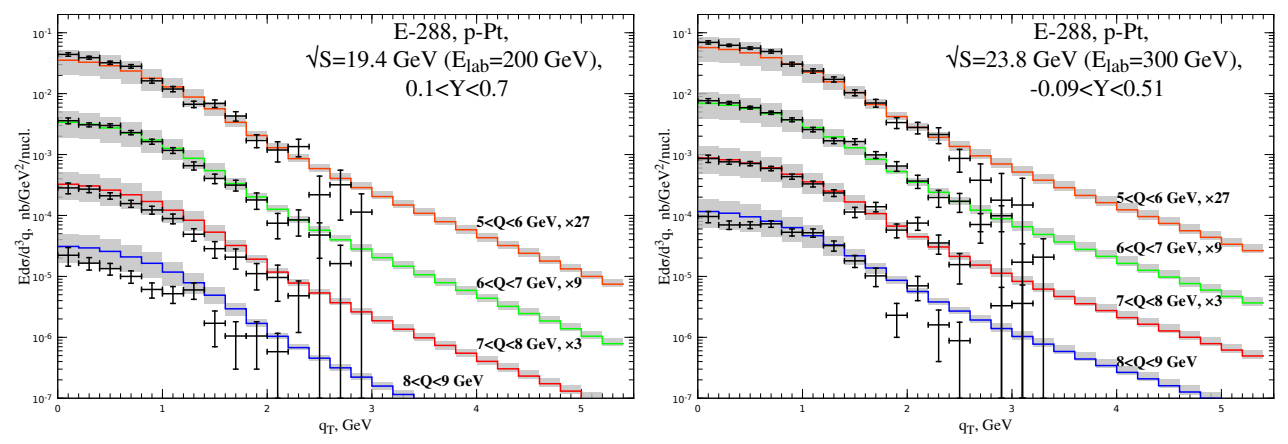

Figure 2. Transverse momentum spectra of DY pairs. The histogram corresponds to calculation in PRA. The data are from the E288 Collaboration [14], left panel: $\sqrt{S}=19.4 \mathrm{GeV}, 0.1<Y<0.7$; right panel: $\sqrt{S}=23.8 \mathrm{GeV},-0.09<Y<0.51$
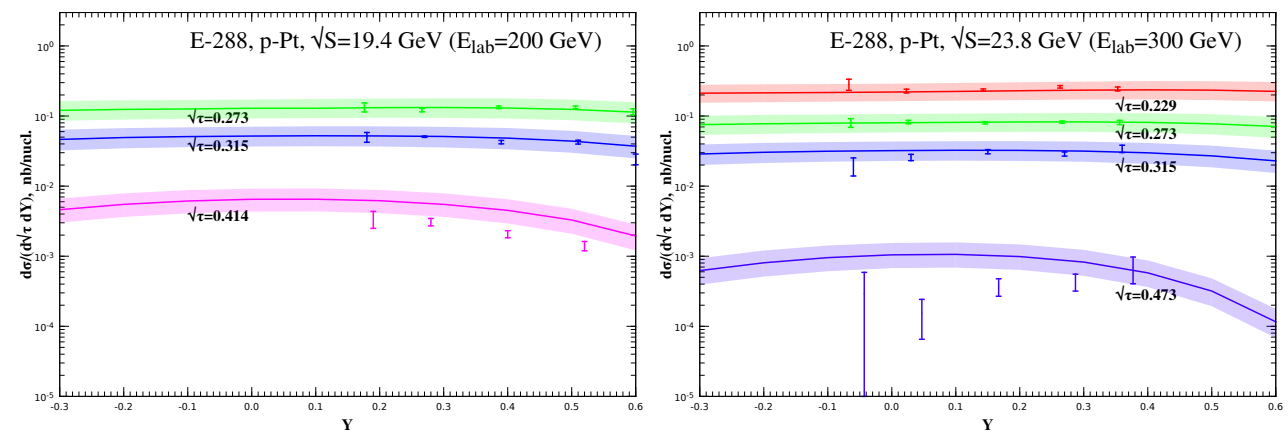

Figure 3. Rapidity spectra of DY pairs. The histogram corresponds to calculation in PRA. The data are from the E288 Collaboration [14], left panel: $\sqrt{S}=19.4 \mathrm{GeV}$; right panel: $\sqrt{S}=23.8 \mathrm{GeV}$

In Fig. 4 the PRA predictions for helicity SFs $F_{U U}^{(1,2, \cos 2 \phi)}$ are plotted for the case of $p p$ collisions with $\sqrt{S}=24 \mathrm{GeV}$ for two bins in the invariant mass of the pair: $2 \leq Q \leq 5 \mathrm{GeV}$ and $5 \leq Q \leq 10 \mathrm{GeV}$.

In the "parton-model style" TMD-factorization [10], based solely on the $q \bar{q}$-annihilation picture (diagram (a) in Fig. 1), the TMD quark correlators for the case of unpolarized protons are parameterized in terms of un-polarized quark distribution $f_{1}^{q}\left(x, \mathbf{q}_{T}^{2}\right)$ and BoerMulders [17] function $h_{1}^{\perp q}\left(x, \mathbf{q}_{T}^{2}\right)$. While the former is responsible for the $\left(1+\cos ^{2} \theta\right)$ angular dependence and contributes mostly to $F_{U U}^{(1)}$ function, the latter leads to nonzero $F_{U U}^{(\cos 2 \phi)}$. The $\left(1-\cos ^{2} \theta\right)$ angular dependence does not arise in TMD-factorization at the leading power in $q_{T}^{2} / Q^{2}$ [10]. In agreement with this, in PRA the $\mathrm{SF} F_{U U}^{(2)}$ is suppressed by factor $Q^{2}$ w.r.t. $F_{U U}^{(1)}$.

As we have shown in [18], the numerical value of $F_{U U}^{\cos 2 \phi}$ even at $q_{T}^{2} \ll Q^{2}$ strongly depends on the details of the procedure of restoration of gauge-invariance of the hard-scattering coefficient. On the other hand, in the TMD-factorization, based on the diagram (a) in Fig. 1, the hadronic tensor (e.g. Eq. (73) in [10]) does not satisfy Ward identity for $q_{T} \neq 0$. This raises serious doubts about the Boer-Mulders function as a well-defined physical quantity in this approach. 

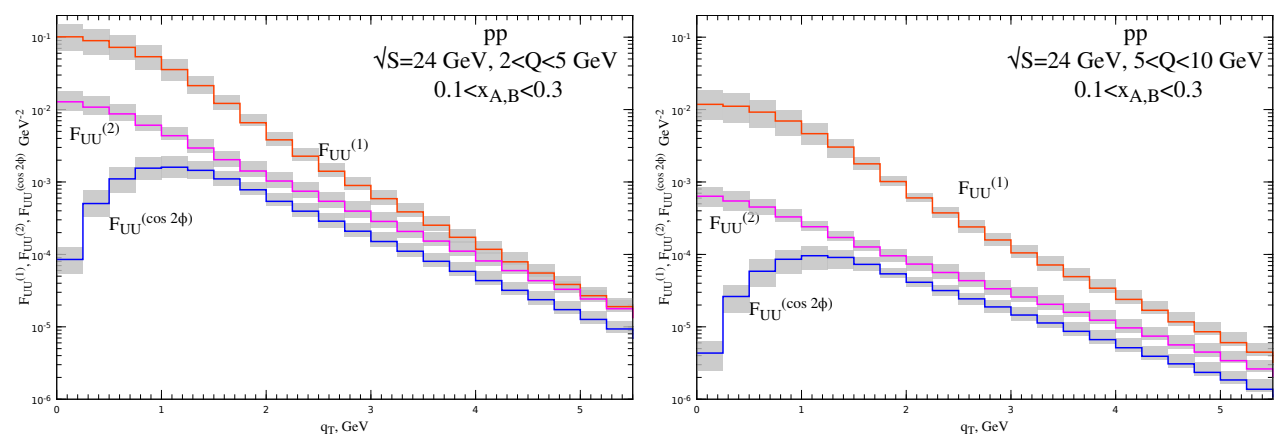

Figure 4. Predictions for unpolarized Drell-Yan SFs $F_{U U}^{(1)}, F_{U U}^{(2)}$ and $F_{U U}^{(\cos 2 \phi)}$ in $p p$-collisions at $\sqrt{S}=24$ $\mathrm{GeV}$. Solid lines with uncertainty bands - PRA predictions

Acknowledgements. Authors thank the Foundation for the Advancement of Theoretical Physics and Mathematics BASIS, grant No. 18-1-1-30-1. The work of M.N. is supported in part by the Russian Foundation for Basic Research through the Grant No. 18-32-00060.

\section{References}

[1] J.C. Collins, D.E. Soper, and G.F. Sterman, Nucl. Phys. B 250, 199 (1985)

[2] J. C. Collins, Foundations of perturbative QCD (Cambridge University Press, Cambridge, 2011)

[3] A.V. Karpishkov, M.A. Nefedov, and V.A. Saleev, Phys. Rev. D 96, 096019 (2017)

[4] L.N. Lipatov, Phys. Rept. 286, 198 (1997)

[5] M.A. Kimber, A.D. Martin, and M.G. Ryskin, Phys. Rev. D 63, 114027 (2001)

[6] G. Watt, A. D. Martin, and M. G. Ryskin. Eur. Phys. J. C 31, 73 (2003)

[7] V.S. Fadin and V.E. Sherman, JETP Lett. 23, 599, (1976)

[8] [13] L.N. Lipatov and M.I. Vyazovsky, Nucl. Phys. B 596, 399 (2001)

[9] I. Balitsky and G.A. Chirilli, Phys. Rev. D 88, 111501 (2013)

[10] S. Arnold, A. Metz, and M. Schlegel. Phys. Rev. D 79, 034005 (2009)

[11] J.C. Collins and D.E. Soper, Phys. Rev. D 16, 2219 (1977)

[12] M. A. Nefedov, N. N. Nikolaev, and V. A. Saleev, Phys. Rev. D 87, 014022 (2013)

[13] I. A. Savin et al., EPJ Web Conf. 85, 02039 (2015)

[14] A. S. Ito et al., D Phys. Rev. 23, 604 (1981)

[15] A.D. Martin, W.J. Stirling, R.S. Thorne, and G. Watt, Eur. Phys. J. C 63, 189 (2009)

[16] L.Y. Zhu et al., Phys. Rev. Lett. 102, 182001 (2009)

[17] D. Boer and P.J. Mulders, Phys. Rev. D 57, 5780 (1998)

[18] M.A. Nefedov and V.A. Saleev, Preprint arXiv:1810.04061 [hep-ph] (2018) 